\title{
Manejo avanzado de la vía aérea en pacientes con sospecha o diagnóstico de COVID-19
}

Advanced airway management in patients with suspected or diagnosed COVID-19

Manejo avançado das vias aéreas em pacientes com suspeita ou diagnóstico de COVID-19

Pamela Mercado Velázquez, ${ }^{*}$ Víctor Acosta Nava, ${ }^{*}$ José Ángel Baltazar Torres, ${ }^{\star}$ José Luis Hernández Oropeza, ${ }^{*}$

Eduardo Rivero Sigarroa, ${ }^{*}$ Guillermo Domínguez Cherit*

\section{RESUMEN}

En marzo de 2020, la Organización Mundial de la Salud (OMS) emitió una alerta de pandemia para la infección por el coronavirus 2 del síndrome respiratorio agudo grave (SARS-CoV-2, por sus siglas en inglés). Este virus produce cuadros clínicos que van desde síntomas respiratorios leves hasta neumonía con criterios de gravedad. La enfermedad producida por el virus SARS-CoV-2 se ha denominado coronavirus disease 19 (COVID-19). El virus SARS-CoV-2 tiene una alta tasa de contagio entre los humanos, lo cual hace que el manejo de los pacientes con COVID-19 implique un riesgo alto de contagio para el personal de salud en el ámbito hospitalario. Por ello, es importante contar con protocolos de manejo estandarizados, que minimicen el riesgo de contagio, sobre todo durante la realización de procedimientos que generen aerosoles, los cuales son considerados como de más alto riesgo. El manejo avanzado de la vía aérea está incluido dentro de esta categoría de procedimientos. El presente documento no tiene la intención de reemplazar las guías actuales sobre el control y manejo estandarizado de las enfermedades infecciosas. Su objetivo es proveer recomendaciones para el manejo avanzado de la vía aérea en este grupo de pacientes, que permitan realizar un procedimiento con seguridad para el enfermo, promover el autocuidado del personal de salud y prevenir la diseminación del virus en el ambiente hospitalario. Palabras clave: Vía aérea, intubación orotraqueal, COVID-19.

\section{ABSTRACT}

In March 2020, the World Health Organization (WHO) issued a pandemic alert for infection with coronavirus 2 of severe acute respiratory syndrome (SARS-CoV-2). This virus produces clinical symptoms that range from mild respiratory symptoms to pneumonia with severity criteria. The disease caused by the SARS-CoV-2 has been called coronavirus disease 19 (COVID-19). The SARS-CoV-2 has a high rate of contagion among humans, which means that the management of patients with COVID-19 implies a high risk of contagion for health personnel in the hospital setting. Therefore, it is important to have standardized management protocols that minimize the risk of contagion, especially during the performance of procedures that generate aerosols, which are considered the highest risk. Advanced airway management is included in this category of procedures. This document is not intended to replace current guidelines on the standardized control and management of infectious diseases. Its objective is to provide recommendations for the advanced management of the airway in this group of patients, which allow performing a procedure safely for the patient, promoting self-care of health personnel and preventing the spread of the virus in the hospital environment. Keywords: Airway, orotracheal intubation, COVID-19.

\section{RESUMO}

Em março de 2020, a Organização Mundial da Saúde (OMS) emitiu um alerta de pandemia pela infecção causada por coronavírus 2 do síndrome respiratória aguda grave (SARS-CoV-2, por suas siglas em inglês). Este vírus produz quadros clínicos que variam de sintomas respiratórios leves a pneumonia com critérios de gravidade. A doença causada pelo vírus SARSCoV-2 foi denominada coronavirus disease 19 (COVID-19). O vírus SARSCoV-2 apresenta alto índice de contágio entre humanos, o que faz com que o manejo de pacientes com COVID-19 implique em alto risco de contágio para profissionais de saúde em ambiente hospitalar. Portanto, é importante ter protocolos de manejo padronizados que minimizem o risco de contágio, principalmente durante procedimentos que geram aerossóis, considerados de

* Instituto Nacional de Ciencias Médicas y Nutrición «Salvador Zubirán», SSA. Ciudad de México, México.

Recibido: 12/05/2020. Aceptado: 07/06/2020.

Citar como: Mercado VP, Acosta NV, Baltazar TJÁ, Hernández OJL, Rivero SE, Domínguez CG. Manejo avanzado de la vía aérea en pacientes con sospecha o diagnóstico de COVID-19. Med Crit. 2021;35(1):47-53. https://dx.doi.org/10.35366/99153

www.medigraphic.com/medicinacritica maior risco. O gerenciamento avançado das vias aéreas está incluído nesta categoria de procedimentos. Este documento não se destina a substituir as diretrizes atuais sobre o controle e gerenciamento padronizados de doenças infecciosas. Seu objetivo é fornecer recomendações para o manejo avançado da via aérea neste grupo de pacientes, que possibilitem a realização de um procedimento com segurança ao paciente, promovam o autocuidado dos profissionais de saúde e evitem a disseminação do vírus no ambiente hospitalar. Palavras-chave: Via aérea, intubação orotraqueal, COVID-19.

\section{INTRODUCCIÓN}

En diciembre de 2019, en Wuhan, China, se reportó una alta tasa de casos de neumonía adquirida en la comunidad. ${ }^{1}$ El 11 de febrero de 2020, el Coronaviridae Study Group (CSG) del International Committee on Taxonomy of Viruses (ICTV), denominó al agente causal de estas infecciones como SARS-CoV-2 y a la enfermedad que produce como COVID-19. ${ }^{2}$ A diferencia de otras infecciones causadas por coronavirus, como SARS-CoV y MERS-CoV, esta enfermedad ha tenido una amplia y rápida diseminación a nivel mundial y en marzo de 2020 fue considerada una pandemia por la OMS. ${ }^{1,3}$ La mortalidad asociada con COVID-19 se ha reportado entre 2 y $3 \%$, la cual es menor que la asociada con SARS-CoV (10-15\%) y a MERS-CoV $(40 \%),{ }^{5}$ pero significativamente mayor que la reportada durante la pandemia de influenza H1N1 en el 2009 (0.023\%). ${ }^{6}$

SARS-CoV-2 es un $\beta$-coronavirus formado por una sola cadena encapsulada de ácido ribonucleico (ARN). ${ }^{7}$ Se cree que su transmisión al humano ocurrió a través de animales hospederos como los murciélagos o los pangolines. ${ }^{2,3}$ Este virus se transmite de humano a humano por medio del tracto respiratorio, a través de gotas (mayores a $5 \mu \mathrm{m}$ ), secreciones respiratorias y por contacto directo con una dosis pequeña. Su periodo de incubación va desde uno a 14 días, aunque habitualmente es de tres a siete días. ${ }^{8}$ Los individuos infectados por SARS-CoV-2 son el principal medio de transmisión, en especial durante la fase de latencia. ${ }^{3}$ Además, se ha descrito que los individuos infectados, pero asintomáticos, pueden jugar un papel crítico en la transmisión del virus. ${ }^{9}$

\section{RIESGO DE CONTAGIO EN EL PERSONAL DE SALUD}

A pesar de los esfuerzos por controlar la transmisión intrahospitalaria de la enfermedad, en esta pandemia 
ya se han reportado casos de infección en el personal de salud. ${ }^{10,11}$ De acuerdo con el Chinese Center for Disease Control and Prevention (CCDC), hasta febrero de 2020 existían más de 3,000 trabajadores de la salud infectados en China. ${ }^{12}$

No se tienen aún cifras exactas del personal de salud que se ha infectado en México durante esta pandemia, pero se estima que los especialistas con mayor riesgo de contraer la infección son los anestesiólogos, intensivistas y urgenciólogos. ${ }^{13,14}$

En 2003, durante el brote de SARS en Ontario, Canadá, $51 \%$ de los casos detectados correspondieron a trabajadores de la salud. Los profesionales de la salud involucrados en la intubación endotraqueal de pacientes infectados tuvieron seis veces más riesgo de adquirir la infección, en comparación con aquellos que no participaron en este procedimiento. ${ }^{15}$

En COVID-19, los procedimientos que generan aerosoles son los principales causantes de contagio en el personal de salud. ${ }^{16}$ La Figura 1 muestra el riesgo de contagio, expresado en odds ratio (OR), de los procedimientos que generan aerosoles. La intubación endotraqueal es el procedimiento que genera más aerosoles y, por lo tanto, es el que mayor riesgo de contagio implica. Se han descrito algunas técnicas que podrían ser útiles para disminuir la exposición a aerosoles durante la intubación endotraqueal, como el uso de una caja contra aerosoles, el uso de un plástico cobertor o la administración de medicamentos intravenosos para disminuir la tos. ${ }^{17,18}$ Sin embargo, la utilidad real de estas técnicas aún se desconoce.

Tanto la exposición prolongada como la pobre adherencia a las medidas de control de infecciones favorecen la adquisición de infecciones ocupacionales. Utilizar espacios de trabajo no adecuados y la falta de equipo

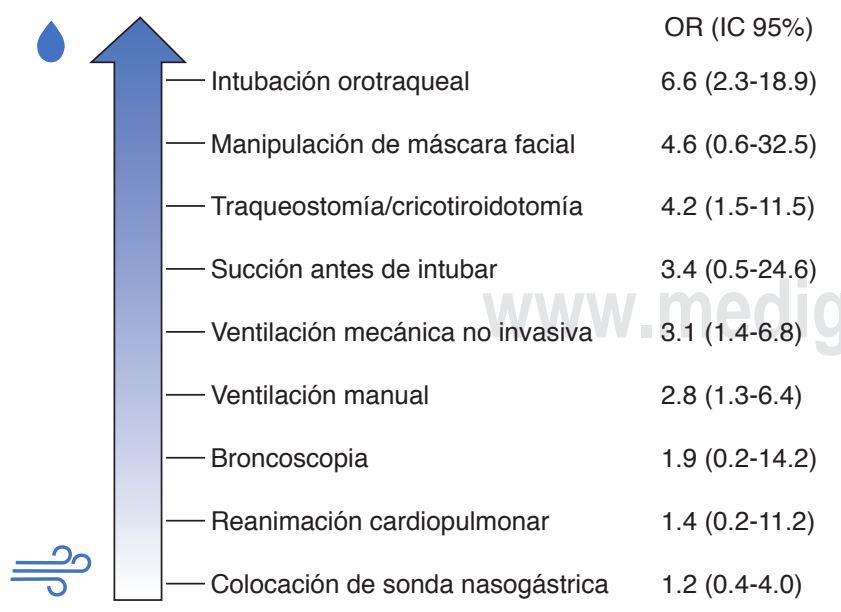

Figura 1: Odds ratio para el riesgo de contagio del personal de salud de los procedimientos generadores de aerosoles.

$\mathrm{OR}=$ odds ratio, IC $95 \%$ = intervalo de confianza del $95 \%$.

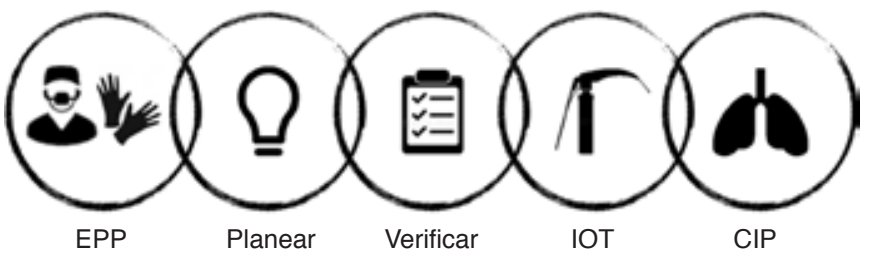

Figura 2: Cadena de intubación orotraqueal en pacientes con sospecha 0 diagnóstico de COVID-19.

$\mathrm{EPP}=$ equipo de protección personal, IOT = intubación orotraqueal, $\mathrm{CIP}=$ cuidados integrados postintubación.

de protección personal (EPP) también contribuyen a la transmisión nosocomial. ${ }^{16}$

Por lo anterior, el personal de salud encargado de la atención de pacientes con COVID-19 debe tener alta conciencia y conocimiento para la realización de estos procedimientos. Debe estar convencido de que el realizarlos implica un claro beneficio sobre el riesgo en la evolución del paciente, que deben ser hechos sólo por personal altamente capacitado para ello y sólo si se cuenta con el EPP completo y en buen estado.

\section{PROTOCOLO PARA EL MANEJO AVANZADO DE LA VÍA AÉREA EN PACIENTES CON COVID-19}

Debido a que la intubación endotraqueal se ha identificado como el procedimiento con mayor riesgo de contagio para el personal de salud, es necesario estandarizar la técnica para que el procedimiento sea seguro, confiable y con la mayor probabilidad de éxito al primer intento. De acuerdo con el Royal College of Anaesthetists y otras asociaciones del Reino Unido, el manejo avanzado de la vía aérea en pacientes con COVID-19 debe ser recordado con el acrónimo SEP (SAS, por sus siglas en inglés). ${ }^{19}$

S Seguro. Para el equipo de salud y para el paciente.

E Exacto. Evitar técnicas repetidas, desconocidas o peligrosas.

P Preciso. En el momento indicado, sin prisa, sin retraso y al primer intento.

Con base en esto, proponemos una cadena de intubación endotraqueal, la cual consiste en una práctica de los elementos necesarios para intubar a un paciente de forma SEP (Figura 2) y que se describe a continuación:

- EPP: el cual debe estar completo y en adecuadas condiciones.

- Planear: todo el equipo involucrado debe tener conocimiento de los pasos a realizar, en especial si el paciente tiene predictores de vía aérea difícil (VAD) o si ya se ha determinado que tiene VAD.

- Verificar: antes de iniciar el procedimiento, todo el material debe estar dentro del cuarto de intubación y 
funcionando adecuadamente, ya que una vez iniciado el procedimiento no es recomendable abrir el cuarto.

- Intubación endotraqueal (IOT): debe ser realizada por personal experto en el manejo avanzado de la vía aérea.

- Cuidados integrados postintubación (CIP).

\section{Equipo de protección personal}

Antes de incorporarse al equipo de atención de los pacientes con COVID-19, el personal de salud debe recibir capacitación y entrenamiento sobre los protocolos de prevención de infecciones, así como de la correcta colocación y retiro del EPP.

EI EPP a utilizar por el personal de salud depende de la actividad que se va a realizar y, por lo tanto, del área donde se encuentre. La Figura 3 muestra un sistema que consiste en clasificar en zonas fría, tibia y caliente las áreas alrededor del paciente infectado y el EPP necesario en cada zona.

El EPP necesario para el manejo avanzado de la vía aérea incluye:

1. Gorro quirúrgico desechable.

2. Lentes de protección con sello completo a la cara (preparados para que no se empañen, ya sea con líquido antiempañante o una gota de jabón).

3. Cubrebocas N95.

4. Careta/escafandra.
5. Pijama quirúrgica exclusiva para este procedimiento.

6. Bata quirúrgica impermeable a líquidos.

7. Dos pares de guantes no estériles, de preferencia de color diferente y largos.

8. Botas quirúrgicas desechables.

La colocación del EPP debe ser supervisada por un observador antes de entrar a las zonas tibia y caliente.

\section{Planear}

Se debe realizar un adecuado plan para intubar al paciente. Es importante que cada miembro del equipo conozca las actividades que debe desempeñar. En todo momento se debe tener una comunicación clara, simple y de circuito cerrado (repetir instrucciones). Este procedimiento debe realizarse idealmente en un cuarto con presión negativa para minimizar la exposición a gotas o aerosoles. Si no se cuenta con presión negativa, el cuarto debe tener las puertas cerradas y se debe apagar el aire acondicionado. Debe realizarse una exploración rápida de la vía aérea utilizando la nemotecnia COVID.

C. Cabeza o cuello lesionados.

O. Objeto suelto en la cavidad oral.

V. Visible cuello ancho.

I. Historia de vía aérea difícil.

D. Distancia tiromentoniana $<6 \mathrm{~cm}$ y/o distancia interincisivos $<3 \mathrm{~cm}$.

Zona caliente (dentro del cubículo del paciente)
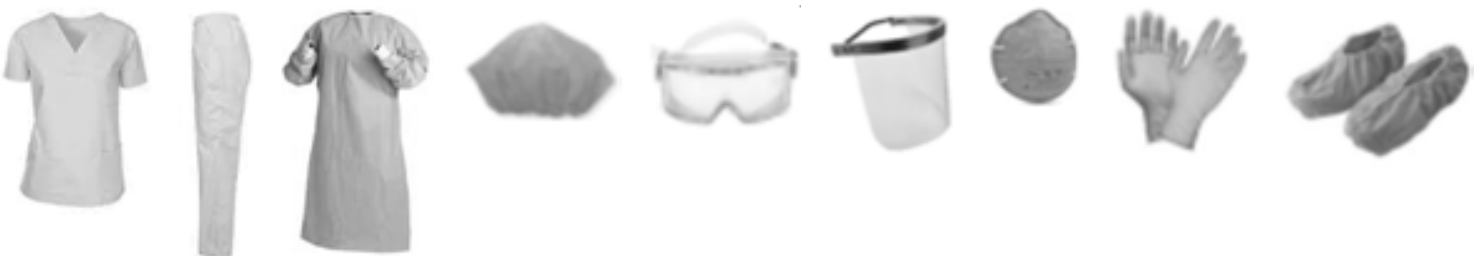

Zona tibia (dentro de la Unidad de Terapia Intensiva)
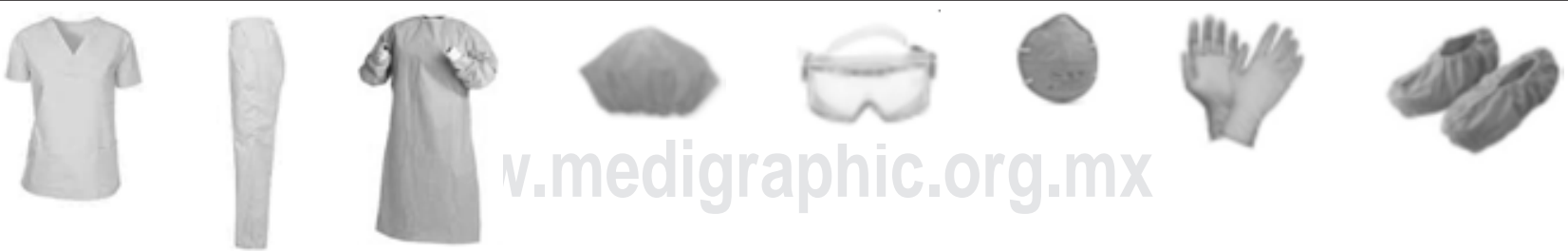

Zona fría (fuera de la Unidad de Terapia Intensiva)

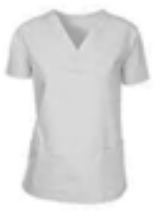

Figura 3:

Equipo de protección personal necesario en cada una de las áreas durante la intubación endotraqueal de pacientes con COVID-19. La careta o escafandra sólo deberá utilizarse durante la producción de aerosoles. 


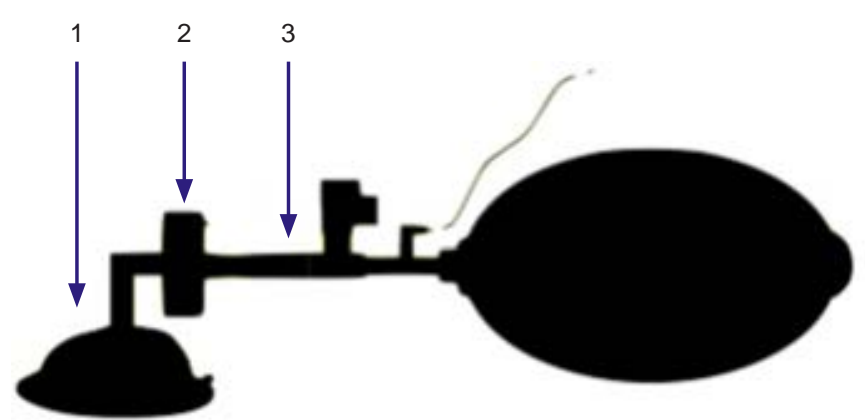

Figura 4: Colocación correcta de la mascarilla facial (1), filtro hidrofílico (2) y circuito de ventilación (3).

No recomendamos realizar exploración de la vía aérea que requiera acercamiento a la cavidad oral del paciente, como sería la evaluación de Mallampati. Sólo debe ingresar al cuarto de intubación el número mínimo necesario de personas para realizar el procedimiento. Recomendamos un operador de la vía aérea, un asistente de vía aérea y una persona para preparar y administrar medicamentos. En total, tres personas dentro del cuarto de intubación. Afuera del cuarto una persona asistente con un carro de paro y equipo para realizar cricotiroidotomía. La persona más experimentada es la que debe realizar la intubación, acompañada de otro médico asistente (anestesiólogo o intensivista), con el objetivo de disminuir el número de intentos para intubar.

\section{Verificar}

Debe corroborarse que todo el material y equipo necesario para realizar el procedimiento esté listo antes de ingresar al cuarto de intubación (mascarilla facial, circuito de ventilación «Mapleson's C», filtro hidrofílico, cánula de succión, medicamentos intravenosos, vía de acceso intravenoso, videolaringoscopio, tubo endotraqueal, guía metálica, cánula de Guedel, jeringa de 20 $\mathrm{cm}^{3}$, máscara laríngea de segunda generación, fijador de tubo endotraqueal, capnógrafo, gasas no estériles, campo estéril y dos bolsas para deshecho). Además, antes de realizar el procedimiento debe instalarse dentro del cuarto de intubación la máquina de ventilación mecánica y el equipo de monitorización (tipo I), con todos sus aditamentos. Es de vital importancia corroborar que el filtro hidrofílico se encuentre colocado entre la mascarilla facial y el circuito de ventilación o la bolsa respiradora, como se muestra en la Figura 4. Debido a que el primer intento debe ser el mejor, es preciso que todos los casos sean intubados utilizando guía metálica y videolaringoscopio, ya que éste minimiza el tiempo de generación y exposición a aerosoles. ${ }^{20}$ Sugerimos que se preparen infusiones de hipnóticos sedantes y analgésicos (midazolam, propofol, fentanilo), así como vasopresores (norepinefrina), antes de que ingrese el paciente al cuarto de intubación. Si en su institución se encuentra disponible, puede utilizar un dispositivo para disminuir la dispersión de aerosoles. Una vez que todo lo anterior se encuentre preparado, debe realizarse una verificación en voz alta, utilizando la lista de verificación que se muestra en la Figura 5.

\section{Intubación orotraqueal}

A continuación, describimos cada uno de los pasos a realizar:

1. Coloque al paciente en la cama del cuarto de intubación.

2. Coloque el sistema de monitoreo (electrocardiografía, oximetría de pulso, baumanómetro, programe la medición de la presión arterial no invasiva cada 3 minutos).

3. Coloque al paciente en posición de olfateo, con elevación de la cabecera a $45^{\circ}$ (rampa/Trendelenburg invertido) a requerimiento del operador.

4. Coloque un campo estéril sobre el hombro izquierdo del paciente.

5. Coloque la caja contra aerosoles con campo desechable cubriendo hacia los pies del paciente.

Hoja de verificación para el manejo avanzado de la vía aérea en pacientes con COVID-19

EPP adecuadamente colocado

Acceso intravenoso permeable

Máscara facial con filtro hidrofílico

Videolaringoscopio/laringoscopio funcional

Máscara laríngea de segunda generación

Succión funcional

Medicamentos para inducción preparados

Medicamentos para sedación preparados

Adecuada colocación de la guía metálica

Ventilador mecánico programado y circuito del ventilador preparado

Material para colocación de sonda enteral

Equipo de fijación para tubo endotraqueal y sonda enteral preparado

Dos bolsas para deshechos en el cuarto de intubación

Campo estéril en el cuarto de intubación

Cuatro gasas no estériles

Figura 5: Hoja de verificación.

$E P P=$ equipo de protección personal. 

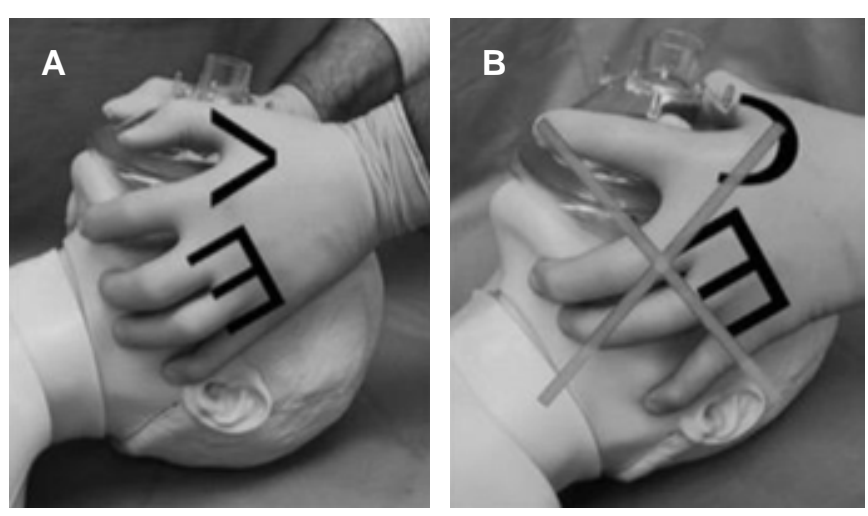

Figura 6: A) Sellado de la mascarilla facial con ambas manos, utilizando la técnica VE. B) La técnica en C debe ser evitada. Tomada de: Cook TM, et al. ${ }^{19}$

6. Cubra la boca y nariz del paciente con gasas húmedas (las gasas no deben bloquear la vía aérea del paciente o quedar dentro de la cavidad oral). ${ }^{13}$

7. Realice preoxigenación con oxígeno al $100 \%$ durante 5 minutos. Para ello, utilice mascarilla facial y mantenga un sello adecuado sobre la cara del paciente (se recomienda realizar sello con las dos manos en posición VE, como se muestra en la Figura 6). ${ }^{19}$

8. Realice secuencia de inducción e intubación rápida (SIIR). La selección de los medicamentos a utilizar depende del estado hemodinámico del enfermo. Sugerimos utilizar el siguiente orden:

a. Lidocaína $1 \mathrm{mg} / \mathrm{kg}$ de peso.

b. Analgésico opioide (fentanilo, sufentanilo, remifentanilo).

c. Hipnótico sedante (propofol, midazolam o considere ketamina 1-2 mg/kg de peso en pacientes hipertensos).

d. Bloqueador neuromuscular (rocuronio $1.2 \mathrm{mg} /$ $\mathrm{kg}$ de peso, succinilcolina $1 \mathrm{mg} / \mathrm{kg}$ de peso).

9. Intente NO ventilar. Si es sumamente necesario, utilice volúmenes pequeños con baja presión.

10. Retire las gasas húmedas y realice la intubación. Obtenga una imagen clara de la glotis (esto puede ser difícil debido a la condensación del vapor generado por el EPP). Corrobore que el tubo endotraqueal pase en medio de las cuerdas vocales y posiciónelo aproximadamente a $1-2 \mathrm{~cm}$ de las cuerdas vocales.

11. Retire el videolaringoscopio de la boca del paciente y colóquelo en una bolsa de deshecho, ciérrela y envíela al área apropiada para su adecuada descontaminación y desinfección. Cubra con un campo estéril los componentes del videolaringoscopio que no sean desechables (cable, cámara, etc.).

12. Infle el globo del tubo endotraqueal con 25-30 $\mathrm{mmHg}$ (no corrobore la presión del globo si no tiene un manómetro específico para ello).
13. Si utilizó guía metálica o algún otro dispositivo (estilete, boogie), retírelo cuidadosamente con una gasa estéril para evitar difundir las secreciones y colóquelo en una bolsa de desecho.

14. Conecte el circuito de ventilación mecánica e inicie el funcionamiento del ventilador mecánico.

15. Verifique la adecuada colocación del tubo endotraqueal mediante capnografía y el movimiento simétrico del tórax (con el EPP resulta difícil la auscultación del tórax y no es recomendable modificar el EPP dentro del cuarto de intubación). Realice radiografía de tórax o ultrasonido de pulmón y vía aérea si tiene duda de la correcta colocación del tubo endotraqueal.

16. Fije el tubo endotraqueal.

\section{Cuidados integrados postintubación}

El coronavirus puede sobrevivir en superficies inertes hasta por nueve días. Sin embargo, el SARS-CoV-2 es susceptible de ser eliminado con alcohol al $62-71 \%$, peróxido de hidrógeno al $0.5 \%$ o hipoclorito se sodio al $0.1 \%{ }^{1}$ Todo el equipo utilizado para el manejo de la vía aérea debe ser descontaminado y desinfectado de modo pertinente. Sugerimos realizarlo en el siguiente orden:

1. La guía metálica de intubación, sonda de succión (Yankauer), cánula de Guedel y hoja desechable de intubación deben colocarse en una bolsa de basura cerrada para su desecho.

2. Desinfecte los guantes externos con solución de alcohol con clorhexidina por 20-30 segundos.

3. Si utilizó caja contra aerosoles, retírela de la cama del paciente y límpiela con cualquiera de las sustancias a las que es susceptible el virus. Posteriormente, repita el paso 2.

4. Retírese los guantes externos de forma lenta y suave para evitar contaminar o rasgar los guantes internos. Utilice la técnica del pico.

5. Desinfecte los guantes internos con solución de alcohol con clorhexidina por 20-30 segundos.

6. Colóquese unos guantes externos nuevos no estériles.

7. Limpie el videolaringoscopio no desechable con toallas de peróxido de hidrógeno al $0.5 \%$ (este paso depende del equipo que se haya utilizado. Recomendamos seguir las instrucciones de limpieza del fabricante).

8. Salga del cuarto de intubación y pida que se realice la limpieza de éste durante al menos 20 minutos.

9. Conecte el videolaringoscopio a la corriente eléctrica.

10. Desinfecte los guantes externos con solución de alcohol con clorhexidina por 20-30 segundos.

11. Vaya a la zona designada para el retiro del EPP. 
Una vez que el paciente se encuentre intubado, se recomienda no desconectar el circuito del ventilador mecánico hasta el momento de la extubación. Si se realiza aspiración traqueal, utilice un circuito cerrado de aspiración. La colocación de una sonda enteral también incrementa el riesgo de contagio, por lo que sugerimos que al término de la intubación, si las condiciones del paciente lo permiten, se coloque en ese momento la sonda enteral.

\section{INTUBACIÓN OROTRAQUEAL EN PACIENTES CON PREDICTORES DE VAD}

En este grupo de pacientes, la intubación con el enfermo despierto es inevitable. La intubación por broncoscopia transnasal con ventilación espontánea, sedación y anestesia local es una técnica recomendada. ${ }^{19} \mathrm{Si}$ ésta falla, se puede realizar intubación con videolaringoscopio, fibrobroncoscopio por cavidad oral o a través de la membrana cricotiroidea con el paciente despierto. Si la probabilidad de fallar con las técnicas anteriores es alta, puede realizarse traqueostomía con apoyo del equipo quirúrgico. ${ }^{21}$

El algoritmo modificado para el manejo avanzado de la vía aérea en pacientes con COVID-19 permite realizar tres intentos de laringoscopia por un médico experto. Si esto falla, se recomienda ventilar al paciente con máscara laríngea de segunda generación y, posteriormente, intubarlo a través de la máscara laríngea o realizar cricotiroidotomía. ${ }^{19}$

\section{CINCO REGLAS BÁSICAS DURANTE EL MANEJO AVANZADO DE LA VÍA AÉREA}

1. No manipule la vía aérea del paciente si el EPP está incompleto o en mal estado. Recuerde que su protección es la prioridad.

2. Minimice el número de personas expuestas durante el procedimiento.

3. Minimice la generación de aerosoles.

4. Maximice la probabilidad de intubación exitosa al primer intento.

5. Mantenga siempre una comunicación clara, simple y fuerte durante el procedimiento.

\section{CONCLUSIONES}

La intubación endotraqueal en las áreas críticas es considerada un procedimiento de alta complejidad y de alto riesgo. La intubación endotraqueal en pacientes con COVID-19 implica un reto mayor para el personal de salud, ya que se suman la incomodidad de portar el EPP y un estrés mental mayor. El trabajo en equipo y la organización de éste son fundamentales para lograr un mejor desempeño en situaciones complejas. Si se tiene un plan de trabajo, se podrá realizar un procedimiento con seguridad para el enfermo, promover el autocuidado del personal de salud y prevenir la diseminación del virus en el ambiente hospitalario. Además, contar con el EPP adecuado y en buen estado, así como su correcto uso, permitirán disminuir el riesgo de contagio del personal de salud durante la realización de este procedimiento en pacientes altamente contagiosos. Recuerde, su seguridad es siempre la mayor prioridad.

\section{REFERENCIAS}

1. World Health Organization. Novel Coronavirus (2019-nCoV). Data as reported by January 30, 2020. [accessed February 2020] Available from: https://www.who.int/docs/default-source/ coronaviruse/situation-reports/20200130-sitrep-10-ncov. pdf?sfvrsn=d0b2e480 2

2. Guo YR, Cao QD, Hong ZS, Tan YY, Chen SD, Jin HJ, et al. The origin, transmission and clinical therapies on coronavirus disease 2019 (COVID-19) outbreak - an update on the status. Mil Med Res. 2020;7(1):11.

3. Lee PI, Hsueh PR. Emerging threats from zoonotic coronavirusesfrom SARS and MERS to 2019-nCoV. J Microbiol Immunol Infect. 2020;53(3):365-367. doi: 10.1016/j.jmii.2020.02.001.

4. Christian MD, Poutanen SM, Loutfy MR, Muller MP, Low DE. Severe acute respiratory syndrome. Clin Infect Dis. 2004;38(10):1420-1427.

5. Majumder MS, Rivers C, Lofgren E, Fisman D. Estimation of MERS-coronavirus reproductive number and case fatality rate for the spring 2014 Saudi Arabia Outbreak: insights from publicly available data. PLoS Curr. 2014. doi: 10.1371/currents.outbreak s.98d2f8f3382d84f390736cd5f5fe133c.

6. Donaldson LJ, Rutter PD, Ellis BM, Greaves FE, Mytton OT, Pebody RG, et al. Mortality from pandemic A/H1N1 2009 influenza in England: public health surveillance study. BMJ. 2009;339:b5213.

7. Zhou P, Yang XL, Wang XG, Hu B, Zhang L, Zhang W, et al. Addendum: a pneumonia outbreak associated with a new coronavirus of probable bat origin. Nature. 2020;588(7836):E6.

8. Li Q, Guan X, Wu P, Wang X, Zhou L, Tong Y, et al. Early transmission dynamics in Wuhan, China, of novel coronavirusinfected pneumonia. N Engl J Med. 2020;382(13):1199-1207.

9. Rothe C, Schunk M, Sothmann P, Bretzel G, Froeschl G, Wallrauch C, et al. Transmission of 2019-nCoV infection from an asymptomatic contact in Germany. N Engl J Med. 2020;382(10):970-971.

10. Buckley C. Chinese Doctor, silenced after warning of outbreak, dies from Coronavirus. The New York Times. [accessed February 2020] Available in: https://www.nytimes.com/2020/02/06/world/ asia/chinese-doctor-Li-Wenliang-coronavirus.html

11. The Novel Coronavirus Pneumonia Emergency Response Epidemiology Team: The epidemiological characteristics of an outbreak of 2019 novel Coronavirus diseases (COVID-19). China CDC Weekly. 2020;2:113-122.

12. Tran K, Cimon K, Severn M, Pessoa-Silva CL, Conly J. Aerosol generating procedures and risk of transmission of acute respiratory infections to healthcare workers: a systematic review. PLoS One. 2012;7(4):e35797.

13. Bowdle A, Munoz-Price LS. Preventing infection of patients and healthcare workers should be the new normal in the era of novel coronavirus epidemics. Anesthesiology. 2020;132(6):1292-1295. doi: 10.1097/ALN.0000000000003295.

14. Canelli R, Connor CW, Gonzalez M, Nozari A, Ortega R. Barrier Enclosure during Endotracheal Intubation. $N$ Engl J Med. 2020;382(20):1957-1958.

15. Chen X, Liu Y, Gong Y, Guo X, Zuo M, Li J, et al. Perioperative management of patients infected with the novel coronavirus: recommendation from the Joint Task Force of the Chinese 
Society of Anesthesiology and the Chinese Association of Anesthesiologists. Anesthesiology. 2020;132(6):1307-1316.

16. Fowler RA, Guest CB, Lapinsky SE, Sibbald WJ, Louie M, Tang $P$, et al. Transmission of severe acute respiratory syndrome during intubation and mechanical ventilation. Am J Respir Crit Care Med. 2004;169(11):1198-1202.

17. Tung A, Fergusson NA, Ng N, Hu V, Dormuth C, Griesdale DEG. Medications to reduce emergence coughing after general anaesthesia with tracheal intubation: a systematic review and network meta-analysis. Br J Anaesth. 2020. doi: 10.1016/j. bja.2019.12.041.

18. Matava CT, Yu J, Denning S. Clear plastic drapes may be effective at limiting aerosolization and droplet spray during extubation: implications for COVID-19. Can J Anaesth. 2020;67(7):902-904.

19. Cook TM, El-Boghdadly K, McGuire B, McNarry AF, Patel A, Higgs $A$. Consensus guidelines for managing the airway in patients with COVID-19: Guidelines from the Difficult Airway Society, the Association of Anaesthetists the Intensive Care Society, the Faculty of Intensive Care Medicine and the Royal College of Anaesthetists. Anaesthesia. 2020;75(6):785-799.
20. Aziz MF, Dillman D, Fu R, Brambrink AM. Comparative effectiveness of the C-MAC video laryngoscope versus direct laryngoscopy in the setting of the predicted difficult airway. Anesthesiology. 2012;116(3):629-636.

21. Apfelbaum JL, Hagberg CA, Caplan RA, Blitt CD, Connis RT, Nickinovich DG, et al. Practice guidelines for management of the difficult airway: an updated report by the American Society of Anesthesiologists Task Force on Management of the Difficult Airway. Anesthesiology. 2013;118(2):251-270.

Correspondencia:

Pamela Mercado Velázquez

Vasco de Quiroga Núm. 207,

Col. Belisario Domínguez Sección 16,

Alcaldía Tlalpan, 14080, Ciudad de México.

Teléfono: 55 5487-0900, ext. 5013

E-mail: alemap_mercado@hotmail.com. 